1993-05-01

\title{
Resolution enhancement of spaceborne scatterometer data
}

David G. Long

david_long@byu.edu

Perry J. Hardin

perry_hardin@byu.edu

Peter T. Whiting

Follow this and additional works at: https://scholarsarchive.byu.edu/facpub

Part of the Electrical and Computer Engineering Commons

\section{Original Publication Citation}

Long, D. G., P. J. Hardin, and P. T. Whiting. "Resolution Enhancement of Spaceborne Scatterometer Data." Geoscience and Remote Sensing, IEEE Transactions on 31.3 (1993): 7-15

\section{BYU ScholarsArchive Citation}

Long, David G.; Hardin, Perry J.; and Whiting, Peter T., "Resolution enhancement of spaceborne scatterometer data" (1993). Faculty Publications. 708.

https://scholarsarchive.byu.edu/facpub/708

This Peer-Reviewed Article is brought to you for free and open access by BYU ScholarsArchive. It has been accepted for inclusion in Faculty Publications by an authorized administrator of BYU ScholarsArchive. For more information, please contact ellen_amatangelo@byu.edu. 


\title{
Resolution Enhancement of Spaceborne Scatterometer Data
}

\author{
David G. Long, Member, IEEE, Perry J. Hardin, Member, IEEE, and Peter T. Whiting
}

\begin{abstract}
Spaceborne wind scatterometers are designed principally to measure radar backscatter from the ocean's surface for the determination of the near-surface wind direction and speed. Although measurements of the radar backscatter are made over land, application of these measurements has been limited primarily to the calibration of the instrument. This has been due in part to the low resolution of the scatterometer measurements (nominally $50 \mathrm{~km}$ for the Seasat-A Scatterometer [SASS]). In this paper we present a method for generating enhanced resolution radar images of the earth's surface using spaceborne scatterometry. The technique is based on a new image reconstruction technique which takes advantage of the spatial overlap in scatterometer measurements made at different times to provide enhanced imaging resolution. We describe the reconstruction algorithm and demonstrate the technique using both simulated and actual SASS measurements. The technique can also be used with ERS-1 scatterometer data. The SASSderived images, which have approximately $4 \mathrm{~km}$ resolution, dramatically illustrate the resolution enhancement capability of the new technique. The technique permits utilization of both historic and contemporary scatterometer data for medium-scale monitoring of vegetation and polar ice. We discuss the tradeoff between imaging noise and resolution inherent in the technique.

The instrument processing method employed by SASS limits the ultimate resolution which can be obtained with our method. However, with minor modifications to future Doppler scatterometer systems (such as the NASA scatterometer [NSCAT] and its follow-on EoS-era scatterometer) imaging resolutions down to 1$2 \mathrm{~km}$ for land/ice and $5-10 \mathrm{~km}$ for wind measurement can be achieved with a moderate increase in downlink bandwidth (from $5 \mathrm{kbps}$ to $700 \mathrm{kpbs}$ ). We describe these modifications and briefly describe some of the applications of this medium-scale $\kappa^{i} u$-band imagery for vegetation studies, hydrology, sea ice mapping, and the study of mesoscale winds.
\end{abstract}

\section{INTRODUCTION}

Spaceborne wind scatterometers are an important element in future remote sensing systems because of their proven ability to make all-weather measurements of vector winds over the ocean, a capability first demonstrated by the Seasat scatterometer (SASS) in 1978 [20]. The scatterometer does not directly measure the wind. Instead, it measures the normalized radar backscatter coefficient $\left(\sigma^{\circ}\right)$ of the surface. The $\sigma^{o}$ measurements of the ocean's surface are then related to the near-surface wind vector via a geophysical model function to infer the wind. While scatterometers make measurements of $\sigma^{o}$ over land, these measurements have been used primarily for calibration of the instrument. It is the application of

Manuscript received April 8, 1992; revised December 4, 1992.

The authors are with Brigham Young University, Department of Electrical and Computer Engineering, Provo, UT 84602

IEEE Log Number 9207386. scatterometer measurements over land and ice that we wish to explore.

The first spaceborne scatterometer, S-193, flew on Skylab. Simple experiments showed that the $13.9 \mathrm{GHz} \sigma^{\circ}$ values over land were sensitive to vegetation cover, surface water, soil moisture, and physiography [21]. In particular, the Amazon rainforest appeared to be homogeneous, azimuthally isotropic, and relatively insensitive to polarization with a small diurnal variation. These characteristics of the Amazon rainforest led to the use of Amazon rainforest measurements for calibration of the SASS $\sigma^{o}$ measurements [1], [3], [4], [12].

The most comprehensive study to-date of scatterometer measurements over land was conducted by Kennett and $\mathrm{Li}$ [13], [14], who examined the global SASS data at a resolution of $1^{\circ}$ by $1^{\circ}$ in a search for additional homogeneous areas for future scatterometer instrument calibration. They found the Amazon and Congo rainforests and various desert regions to be remarkably uniform and homogeneous and thus deemed them to be the most suitable targets for future scatterometer calibration activities.

Spaceborne wind scatterometers observe $\sigma^{\circ}$ over a wide range of incidence angles. Over land, $\sigma^{\circ}$ may be modeled as a linear function of the measurement incidence angle [4], [13], e.g.,

$$
10 \log _{10} \sigma^{\circ}(\theta)=\mathcal{A}+\mathcal{B}\left(\theta-40^{\circ}\right)
$$

where $\theta$ is the incidence angle of the observation $\left(20^{\circ}<\right.$ $\theta<60^{\circ}$ ) and $\mathcal{A}$ and $\mathcal{B}$ are constants dependent only on the observed surface characteristics. $\mathcal{A}$ is the value of $\sigma^{\circ}$ at an incidence angle of $40^{\circ}$ and can thus be considered the "incidence angle-normalized" $\sigma^{\circ}$. Because of the wide range of measurement incidence angles used by wind scatterometers, the $\mathcal{A}$ and $\mathcal{B}$ coefficients of this model are more useful in common application than the $\sigma^{o}$ measurements directly. The $\mathcal{A}$ and $\mathcal{B}$ coefficients exhibit a remarkable correlation with land type and vegetation cover with typical values of $-0.1 \leq \mathcal{B} \leq-0.22$ and $-40 \leq \mathcal{A} \leq 0$ depending on the surface type and vegetation cover [13], [14].

The coarse resolution (nominally $50 \mathrm{~km}$ ) of the scatterometer measurements, while suitable for ocean wind measurement, is a significant limitation to the application of scatterometer data in land and ice studies. To ameliorate this difficulty we have developed a new method for obtaining enhanced resolution measurements of $\mathcal{A}$ and $\mathcal{B}$ from spaceborne scatterometer measurements. The technique can achieve even higher resolution for suitably modified future scatterometers. This paper describes our approach and provides sample results 
for SASS data. The resulting medium-scale resolution is useful in a variety of studies of land cover and ice. Several successful experiments in vegetation discrimination are summarized in separate paper [18].

Our technique is primarily based on ground-based signal processing. We take advantage of the spatial overlap of the backscatter measurements taken at different times over a given region, and, using an indirect measurement (i.e., reconstruction) formulation, extend the effective resolution. As we shall see, the improved resolution is not without cost: the noise in the $\mathcal{A}$ and $\mathcal{B}$ images increases as we attempt to improve the resolution.

This paper is organized as follows: In order to appreciate the method we first provide background in scatterometry theory and briefly describe the SASS measurement geometry. Next, we develop the measurement model and theory underlying our resolution enhancement technique and describe the estimation algorithm used to obtain the enhanced resolution measurements of $\mathcal{A}$ and $\mathcal{B}$ from the low resolution $\sigma^{\circ}$ measurements. We demonstrate the technique using simulated SASS $\sigma^{\circ}$ measurements of a synthetic scene and then apply the technique to actual SASS measurements of a portion of the Guiana Highlands of South America. We then describe modifications to future NSCAT-class scatterometers to generate even higher resolution imagery using the method and outline potential applications of the enhanced resolution imagery. Finally, we summarize our results. In a separate paper [18] we present enhanced resolution images of the extended Amazon basin and describe successful experiments in vegetation classification using the enhanced resolution images.

\section{SPACEBORNE SCATTEROMETRY}

A spaceborne scatterometer is a radar instrument designed to accurately measure the radar backscatter of the earth's surface from space. Wind scatterometers (such as SASS and ERS1) are specifically designed to make several measurements of $\sigma^{o}$ at a single point from different azimuth angles. These measurements are used to estimate the near-surface wind vector using a geophysical model relating $\sigma^{o}$ to wind velocity (see [17], [19], [22]).

The scatterometer transmits microwave pulses to the surface and measures the backscattered power. Because the backscatter power measurement is noisy, a second measurement of the noise-only power, $P_{N}$, is made. This is subtracted from the signal+noise measurement to estimate the backscattered signal power, $P_{S}$. The normalized radar cross-section $\left(\sigma^{\circ}\right)$ of the surface is then computed using the radar equation, [3], [12], [22]

$$
\sigma_{o}=\frac{(4 \pi)^{3} R^{4} L}{P_{t} G^{2} \lambda^{2} A} P_{S}
$$

where $R$ is the slant range to the surface, $P_{t}$ is the transmitted power, $P_{S}$ is the received backscattered power, $L$ represents known system losses, $G$ is antenna gain, $A$ is the effective illuminated area, and $\lambda$ is the wavelength of the transmitted radiation. $R, G$, and $A$ depend on the measurement geometry.

Since the measurement of $P_{S}$ is noisy, the retrieved $\sigma^{\circ}$ "measurement" will also be noisy. Let $z$ be the noisy measure- ment of $\sigma^{o}$. Then, $z$ will have a variance which is quadratically dependent on the measurement signal-to-noise ratio (SNR). For SASS the normalized standard deviation $K_{p}$ of $z$ can be expressed (approximately) as [9]

$$
K_{p}=\frac{\sqrt{\operatorname{Var}[z]}}{\operatorname{Mean}[z]}=\frac{1}{\sqrt{N_{p} T_{p} B_{c}}}\left\{1+\frac{2}{\mathrm{SNR}}+\frac{1}{\mathrm{SNR}^{2}}\right\}^{1 / 2}
$$

where SNR SNR $=P_{S} / P_{N}$ is the measurement signal-tonoise ratio, $N_{p}$ is the number of pulses integrated into a single $\sigma^{o}$ measurement, $T_{p}$ is the length of each pulse, and $B_{c}$ is the bandwidth of the measurement resolution cell. For SASS, $-20 \mathrm{~dB}<S N R<20 \mathrm{~dB}$ depending on the incidence angle and surface characteristics, $N_{p}=25, T_{p}=5.7 \mathrm{~ms}$, and $2 \mathrm{kHz}$ $\leq B_{c} \leq 20 \mathrm{kHz}$.

The $\sigma^{\sigma}$ measurements also contain uncertainty (which will be treated as additional noise) due to uncertainties in the spacecraft attitude. Combining this uncertainty with (3), the measurement $K_{p}$ can be expressed as

$$
K_{p}=\alpha \sigma^{o 2}+\beta \sigma^{o}+\gamma
$$

where $\alpha, \beta$, and $\gamma$ are known constants which depend on the measurement geometry (via the radar equation and the SNR) as well as the instrument parameters (e.g., $T_{p}, N_{p}$ ) and calibration accuracy.

\section{A. The Seasat Scatterometer (SASS)}

Since our technique is demonstrated using SASS data it is relevant to briefly review the SASS $\sigma^{\circ}$ measurement scheme. SASS used multiple antennas to make $\sigma^{\circ}$ measurements at different azimuth angles. A diagram of the SASS antenna illumination pattern is illustrated in Fig. 1. Each antenna produced an instantaneous footprint several hundred kilometers long and a few kilometers wide. Two sets of two antennas were used, each set illuminating a wide swath on one side of the subsatellite track. The antennas on each side were arranged at two different azimuth angles. For each orbit, these antennas swept a nominally $500 \mathrm{~km}$ wide swath (on each side) which was further resolved into smaller observation cells by means of timing and Doppler filtering as discussed below. Each antenna could operate at both horizontal and vertical polarization. Thus there were four antennas and eight "beams." SASS also made near-nadir $\sigma^{\circ}$ measurements; however, because these measurements have small incidence angles they are not used in our imaging approach.

In the following subsections the SASS measurement cell resolution and measurement cell geometry are described. It is the overlap in the measurement cells from measurements made at different times which is exploited to yield enhanced resolution estimates of $\mathcal{A}$ and $\mathcal{B}$.

\section{B. Measurement Cell Resolution}

SASS and its follow-on mission NSCAT [19] achieve alongtrack resolution by a combination of a narrow antenna pattern and the timing of transmit pulses [12]. Cross-track resolution is obtained by Doppler filtering, the narrow beam pattern, and the antenna illumination pattern azimuth angle geometry. 


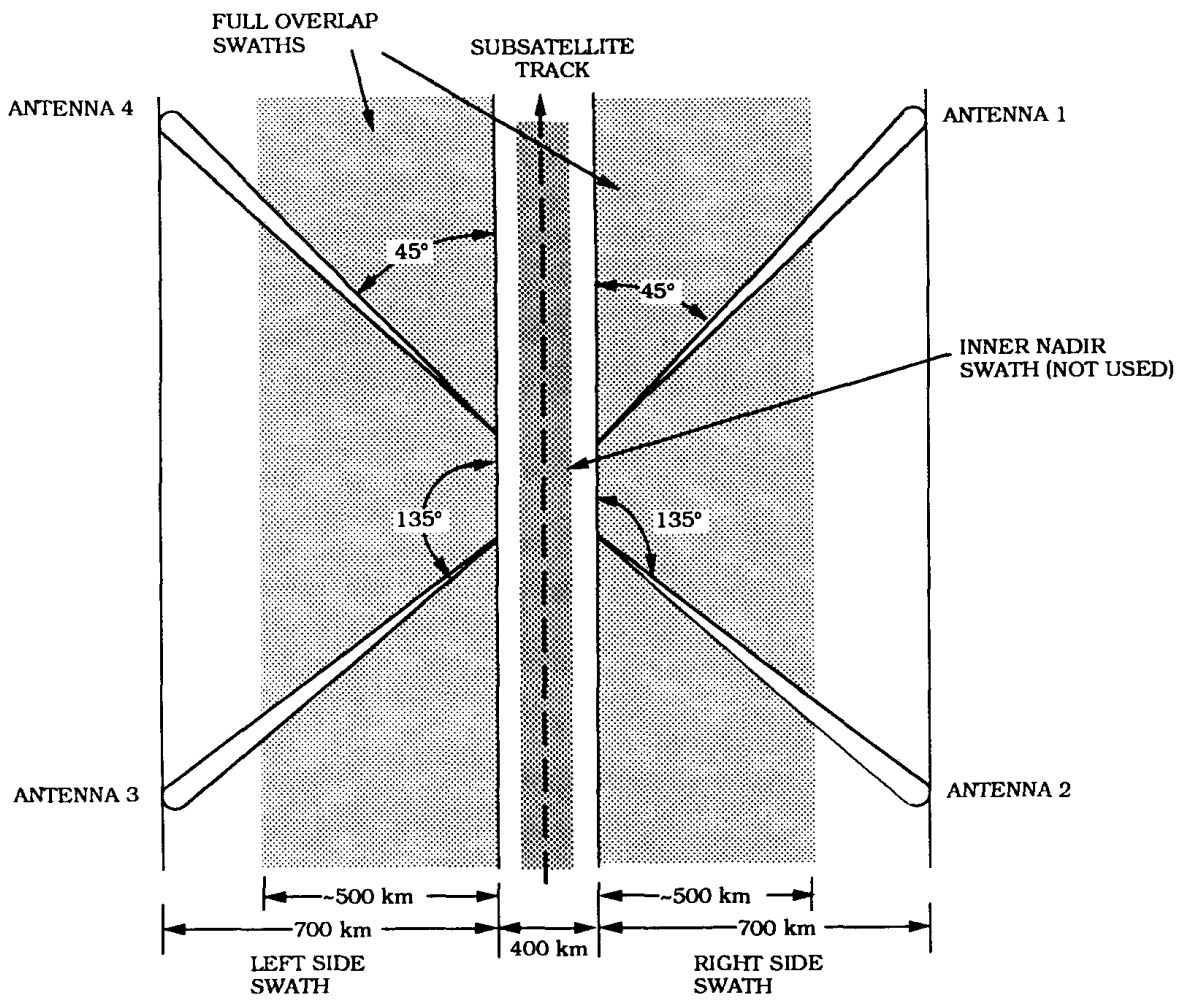

Fig. 1. The Seasat scatterometer antenna illumination pattern. The wind estimate swath is covered by both fore- and aft-facing antennas while only one antenna covers the extended swath width. The nadir swath measurements are not used in the reconstruction method described in this paper.

Along-Beam Resolution: Doppler filtering was used to achieve along-beam resolution for SASS and is planned for future U.S. scatterometers (ERS-1 uses a different technique known as range-gating). Doppler filtering makes use of the fact that the radar echo reflected from the ocean surface is Dopplershifted due to motion of the spacecraft relative to the earth's surface. The return echoes from different portions of the antenna footprint have different Doppler shifts, with a larger shift at far swath and a smaller shift at near swath. This difference in Doppler shift can be exploited to filter the returned echo into cross-track (along-beam) resolution elements or "cells." By processing the returned signal with 15 bandpass filters of unequal bandwidth, SASS resolved the coverage swath on each side of its subsatellite track into 12 cells each having approximately $50 \mathrm{~km}$ spatial resolution. These filters had fixed center frequencies which could not compensate for changing Doppler due to the effects of the earth's rotation resulting in elongation or compression of the cell as the spacecraft moves along its orbit. Thus the measurement cells from different antennas had different resolution and variable area and relative location depending on the position of the spacecraft in the orbit.

Along-Track Resolution: Measurement timing was used to achieve along-track resolution such that the centers of cells measured by each antenna beam were spaced approximately $50 \mathrm{~km}$ apart in the along-track direction. The desired spacing was achieved by making measurements at each azimuth angle once every 6.4 seconds, the time it took for the subsatellite point to move $50 \mathrm{~km}$. During this interval a series of 64 transmit pulses were issued and received for each beam in sequence. In normal operation only four of the eight beams were used. The primary mode employed was dual-sided in which a single polarization (vertical) for each antenna was used. The instrument was also operated less frequently in a single-side mode in which both polarizations from the antennas on a single side of the spacecraft were used.

\section{Measurement Cell Geometry}

The intersection of the Doppler filter bandwidth and the narrow antenna gain pattern define the instantaneous cell resolution for a single pulse. The resulting response can be approximated by the intersection of the $3 \mathrm{~dB}$ antenna beamwidth and the $3 \mathrm{~dB}$ filter response (see Fig. 2). The echo return of 61 transmit pulses were summed into a single integrated $\sigma^{o}$ measurement (the remaining 3 were used to set the receiver gain). Since the spacecraft was moving during these measurements, the integrated measurement cell resolution is (approximately) a six-sided polygon as illustrated in Fig. 3. The dimensions of this integrated cell are a function of the Doppler filter bandwidth, the antenna beamwidth at the surface, the transmit pulse timing, the spacecraft ground track 


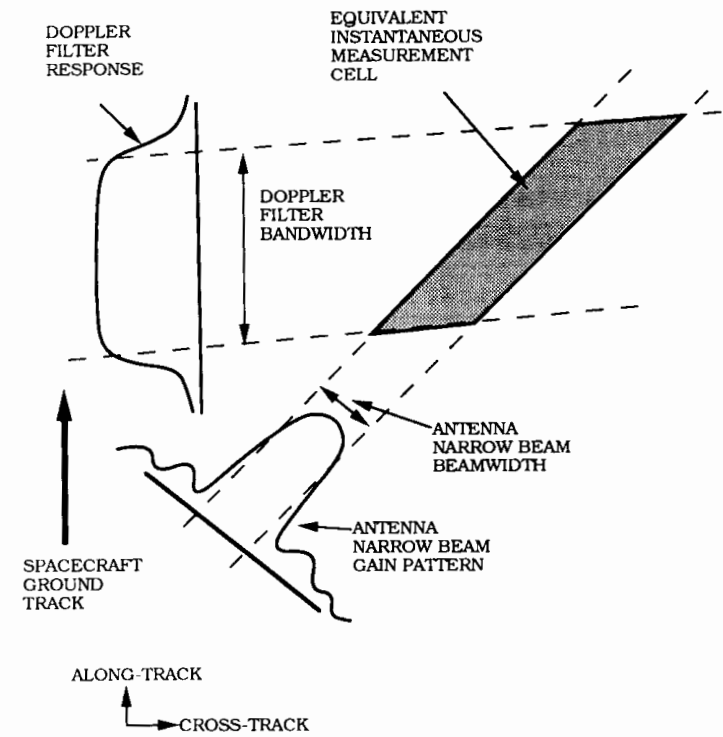

Fig. 2. The combination of the Doppler filters and the narrow antenna gain pattern combine to provide along-beam resolution for a single transmit pulse (corresponding to an instantaneous measurement cell). The actual response is approximated by the $3 \mathrm{~dB}$ equivalent cell area. (Adapted from [12].)

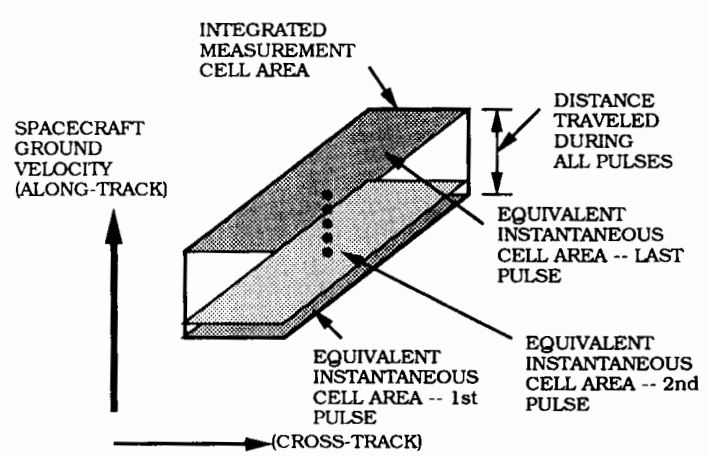

(a)

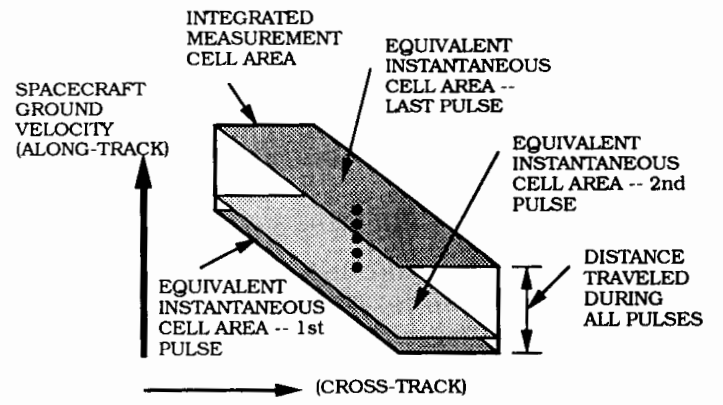

(b)

Fig. 3. Typical six-sided integrated $\sigma^{\circ}$ resolution cells for antennas on the right side of the SASS swath. The cells shown are for the inner swath. The cells become elongated in the outer swath (refer to Fig. 4). (a) Cell from forward-facing antenna. (b) Cell from aft-facing antenna.

velocity, and the cell latitude (see Fig. 9). In this approximate integrated resolution cell, regions outside of the cell area do not contribute to the total $\sigma^{\circ}$ value.

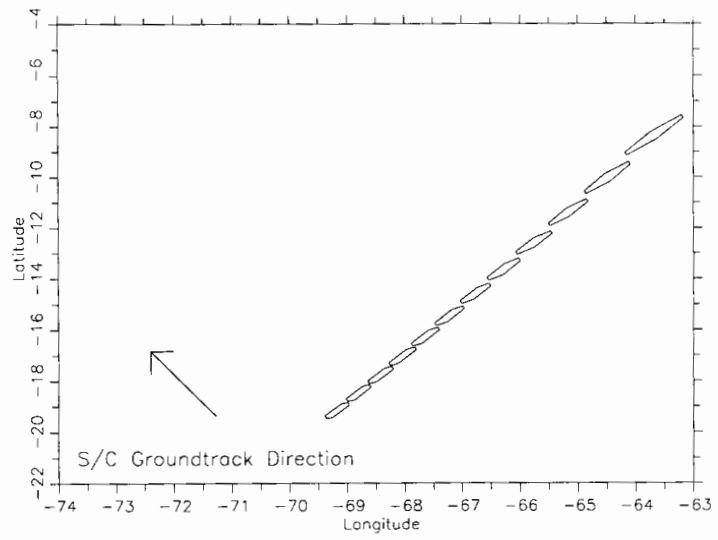

(a)

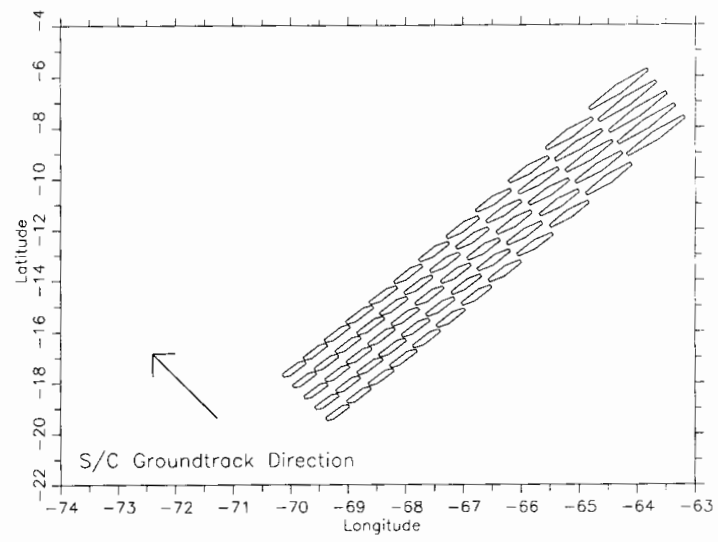

(b)

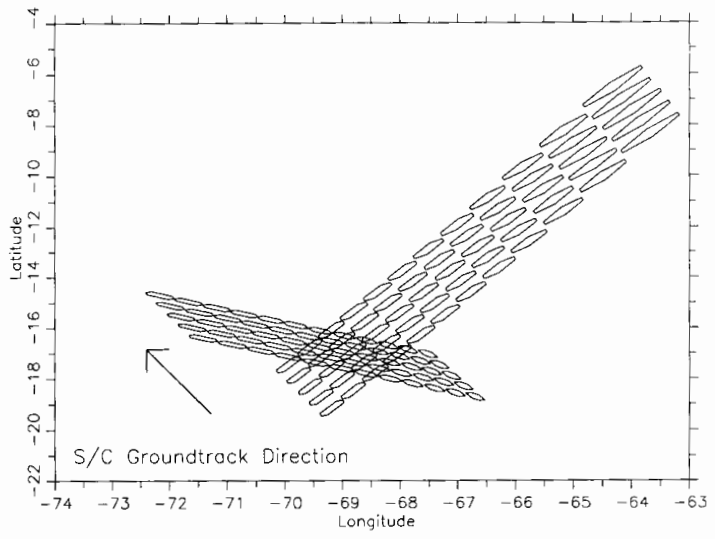

(c)

Fig. 4. (a) Typical layout of SASS integrated $\sigma^{\circ}$ measurement cells for one antenna and a single antenna measurement cycle. Nadir cells are not shown. (b) Positions of $\sigma^{o}$ measurement cells from a single antenna for five antenna cycles depicting the along-track spacing. (c) Positions of $\sigma^{\circ}$ measurement cells from both the fore- and aft-facing antennas illustrating therefore/aft cell overlap and the different along-beam layouts.

Fig. 4(a) illustrates the layout of the measurement cells for a single measurement cycle from a forward-looking antenna on the right side. Fig. 4(b) illustrates the positioning of the 
$\sigma^{o}$ resolution cells of a single antenna for several antenna measurement cycles. As the spacecraft moves along the ground track the measurement cells from the aft antenna overlap the forward antenna as shown in Fig. 4(c). Every few orbits, the spacecraft will pass over this region again on either a northbound (ascending) or a south-bound (descending) ground track (see Fig. 5). This results in criss-cross measurement swaths containing multiple overlapping measurements. For the first half of the mission, the orbit precession shifted the pattern longitudinally which effectively provided coverage of the entire earth's surface. However, during the last half of the mission, the spacecraft operated in an exact-repeat orbit which resulted in diamond-shaped areas which were not observed (refer to [18]).

\section{The Resolution EnHANCEMENT MEASUREMENT METHOD}

In this section, our method for obtaining enhanced resolution radar images of $\mathcal{A}$ and $\mathcal{B}$ in (1) from the low resolution SASS measurements is described. The method is based on the spatial overlap of the $\sigma^{o}$ measurements from multiple SASS passes and on image reconstruction techniques. In order to develop the technique we first describe our enhanced resolution measurement model. We consider the effects of the scatterometer measurement noise and then describe methods for resolution enhancement of $\mathcal{A}$ and $\mathcal{B}$.

\section{A. Enhanced Resolution $\sigma^{\circ}$ Measurement Model}

Consider a rectilinear grid of resolution elements on the earth's surface with a resolution element size of $S_{c} \times S_{a}$. (The subscript $c$ indicates a cross-track dimension while $a$ indicates an along-track dimension.) The six-sided integrated resolution cells of the scatterometer measurements are imposed on this small-scale grid of resolution elements (refer to Fig. 6). Assuming a noise-free measurement, the value of $\sigma^{\circ}$ measured by the scatterometer (denoted by $\varrho_{k}$ where $k$ is the measurement number) will be a weighted average of the $\sigma^{o}$ 's of the individual resolution elements covered by the measurement cell, i.e.,

$$
\varrho_{k}=\sum_{c=L_{k}}^{R_{k}} \sum_{a=B_{k}}^{T_{k}} h(c, a ; k) \sigma^{o}(c, a ; k)
$$

where $L_{k}, R_{k}, T_{k}$, and $B_{k}$ define a bounding rectangle for the $k^{\text {th }}$ hexagonal $\sigma^{o}$ measurement cell, $h(c, a ; k)$ is the weighting function for the $(c, a)^{t h}$ resolution element $(0 \leq$ $h(c, a ; k) \leq 1)$, and $\sigma^{o}(c, a ; k)$ is the $\sigma^{o}$ value for the $(c, a)^{t h}$ resolution element. The incidence angle dependence of $\sigma^{\circ}$ and $h$ is subsumed in the $k$ index. (Over a given scatterometer measurement cell the incidence angle $\theta$ is approximately constant.) $h$ is a function of the cell location and shape.

The dependence of $\sigma^{\circ}$ on $\theta$ can be expressed as (see (1))

$$
\sigma^{\circ}(\theta)=\alpha_{o}^{\mathcal{A}}\left[\beta_{o}(\theta)\right]^{\mathcal{B}}
$$

with

$$
\begin{aligned}
\alpha_{o} & =10^{1 / 10} \\
\beta_{o}(\theta) & =10^{(40-\theta) / 10} .
\end{aligned}
$$

Using this relationship (5) can be written as

$$
\varrho_{k}=\sum_{c=L_{k}}^{R_{k}} \sum_{a=B_{k}}^{T_{k}} h(c, a ; k) \alpha_{o}^{\mathcal{A}(c, a)}\left[\beta_{o}\left(\theta_{k}\right)\right]^{\mathcal{B}(c, a)} .
$$

The actual scatterometer measurements are noisy. Let $z_{k}$ denote the noisy measurement of $\varrho_{k}$, then

$$
z_{k}=\varrho_{k}+\nu_{k}
$$

where $\nu_{k}$ is a zero-mean Gaussian random variable with variance

$$
\operatorname{Var}\left[\nu_{k}\right]=\alpha \varrho_{k}^{2}+\beta \varrho_{k}+\gamma
$$

where $\alpha, \beta$, and $\gamma$ are from the $K_{p}$ equation (4).

The resolution enhancement problem can then be posed as the following reconstruction problem: Given noisy measurements $z_{k}$ of $\varrho_{k}$ for $k \in[1, N]$, determine $\mathcal{A}(c, a)$ and $\mathcal{B}(c, a)$ for each element of the enhanced resolution grid. Our method for solving this problem is described in the next section.

For later use, if we assume that $\mathcal{B}(c, a)$ is known, then (9) can be expressed as

$$
z_{k}=\sum_{c=L_{k}}^{R_{k}} \sum_{a=B_{k}}^{T_{k}} h^{\prime}(c, a ; k) A(c, a)
$$

where

$$
\begin{aligned}
h^{\prime}(c, a ; k) & =h(c, a ; k)\left[\beta_{o}\left(\theta_{k}\right)\right]^{\mathcal{B}(c, a)} \\
A(c, a) & =\alpha_{o}^{\mathcal{A}(c, a)} .
\end{aligned}
$$

In this case, we estimate $A(c, a)$ and compute $\mathcal{A}(c, a)$ using (14).

\section{B. Application to SASS}

The key to successful resolution enhancement of $\mathcal{A}(c, a)$ and $\mathcal{B}(c, a)$ is taking advantage of the overlap in multiple measurements of the same general region. As will be discussed further, the ultimate $\mathcal{A}$ and $\mathcal{B}$ image resolutions are determined by this measurement overlap. While SASS measurements from the fore- and aft-facing antennas in a single orbit provide some measurement overlap, this overlap is insufficient to adequately apply the technique; hence, data from multiple orbits must be used. As discussed later, this requirement may be relaxed for future Doppler scatterometers.

In order to use data from multiple orbits we must assume the radar characteristics of the target region remain constant for each pass. Additionally, we assume that $\mathcal{A}$ and $\mathcal{B}$ have no azimuthal dependence. These and other requirements and assumptions needed for SASS are explored more fully in a separate paper [18].

The SASS Sensor Data Record (SDR) files used in this study are described in detail in [2]. While the SASS SDR files do not include the integrated cell corners, we developed an algorithm for reconstructing the cell geometry from data available in the SDR files. The SDR files contain $\sigma^{\circ}$ measurements spanning an incidence angle range of $0^{\circ}$ to $70^{\circ}$ and include estimates of the normalized standard deviation $\left(K_{p}\right)$ of the noise for each $\sigma^{o}$ measurement. To minimize the effects of noise in the 

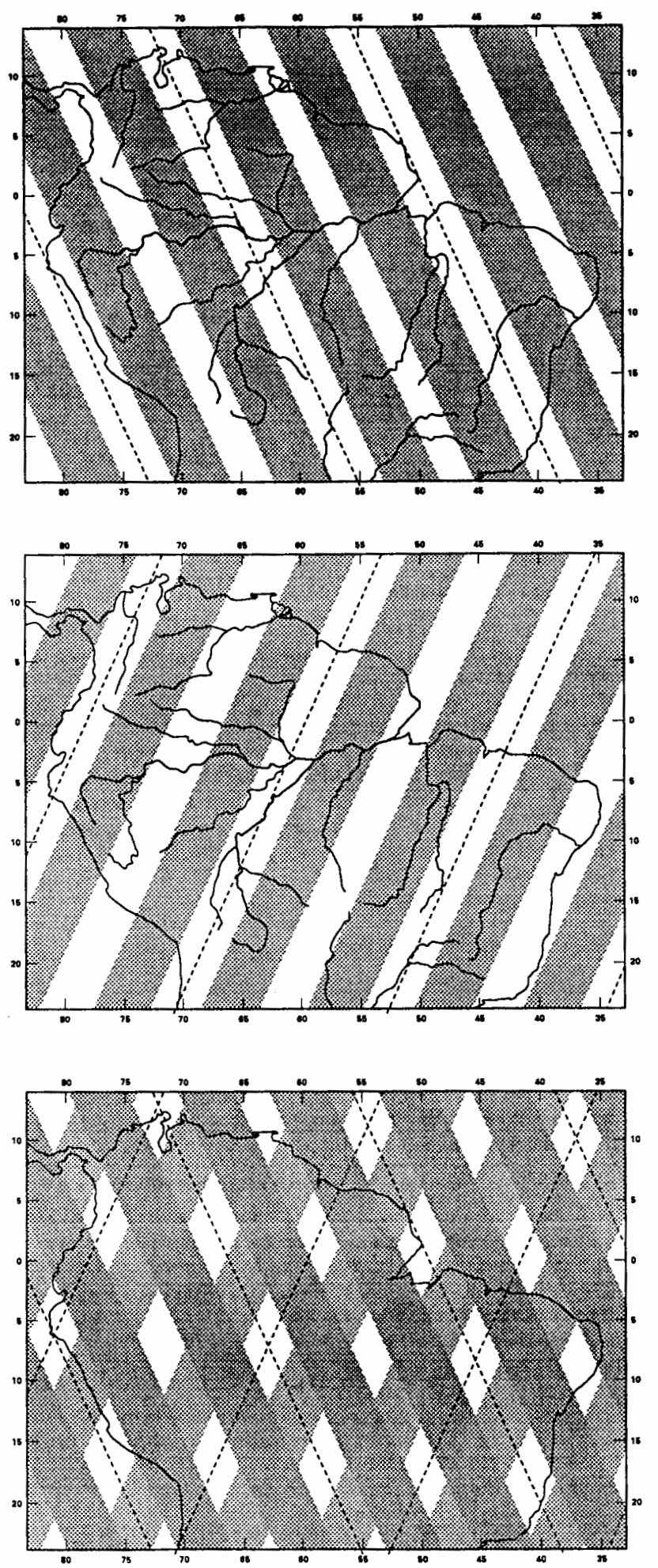

Fig. 5. SASS measurement swath coverage (spanning several days) showing the crossing patterns of ascending and descending orbit passes. The swaths shown are for measurements having incidence angles between $23^{\circ}$ and $57^{\circ}$. (a) Ascending passes (north-bound) only. (b) Descending passes (south-bound) only, (c) All passes. 


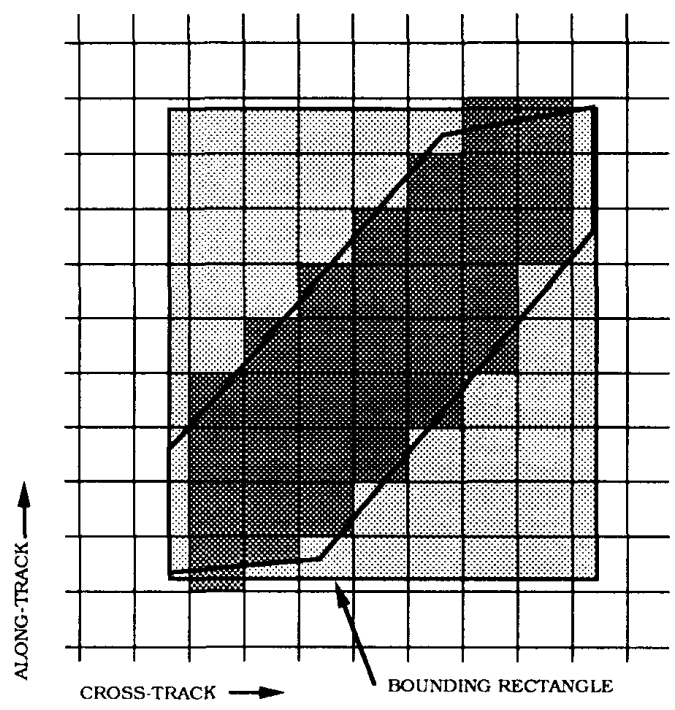

Fig. 6. An integrated SASS $\sigma^{\circ}$ cell overlaying the high resolution grid. Only the shaded square grid elements have nonzero $h(c, a ; k)$. The bounding rectangle is also indicated.

images, $\sigma^{o}$ measurements with excessive $K_{p}\left(K_{p}>15 \%\right)$ were excluded from the reconstruction given below. The discarded measurements, which primarily occurred in the far swath at large incidence angles, represent less than $5 \%$ of the land $\sigma^{\circ}$ measurements. Bracalente and Sweet [4] observed that the linear model in (1) is applicable for measurements only within the incidence angle range of $23^{\circ}$ to $57^{\circ}$ with an error less than $0.5 \mathrm{~dB}$; hence, only $\sigma^{\circ}$ measurements made with incidence angles in the range $23^{\circ}$ to $57^{\circ}$ are used.

\section{Reconstruction Approach}

In order to develop methods for estimation of $\mathcal{A}$ and $\mathcal{B}$ on the enhanced resolution grid, we first examine the case when $\mathcal{B}(c, a)$ is known. Consider an $N_{c} \times N_{a}$ element region of the $S_{c} \times S_{a}$ resolution grid. Collecting all the measurements $z_{k}$ whose bounding rectangles are completely contained within the region of interest, we obtain a matrix equation relating the measurements (in $Z$ ) to $A(c, a)$ (in $S$ ) (refer to $(10)$ and (12)-(14))

$$
Z=H S+\mathcal{V}
$$

where $Z$ is an $N$-dimensional vector of the measurements $z_{k}$, $H$ is an $N \times M\left(M=N_{c} N_{a}\right)$ matrix containing the $h(c, a ; k)$, $S$ is an $M$-dimensional vector of $A(c, a)$ in lexicographic order (i.e., $s_{n}=A(c, a)$ where $\left.n=c+a N_{c}\right)$, and $\mathcal{V}$ is an $N$ dimensional vector of the noise terms $\nu_{k}$. Depending on the resolution element size and the number of measurements $z_{k}$, $H$ can be either over- or underdetermined.

The elements of the matrix $H$ are computed by determining the intersection of the six-sided integrated $\sigma^{\circ}$ measurements and the resolution elements (refer to Fig. 6). For appropriately sized resolution elements, we can assume that the entries of $H$ are either 1 or 0 . The resulting $H$ matrix is very sparse, though it may be extremely large.
With this formulation, the problem of obtaining estimates of $\mathcal{A}(c, a)$ is reduced to the following problem: given $Z$, we wish to estimate $S$ using (14). $\mathcal{A}(c, a)$ is then computed from the $A(c, a)$ in $S$ using (14).

This problem is similar to classic image reconstruction problems in signal and image processing; however, after extensive experimentation, we found that conventional iterative image reconstruction techniques such as algebraic reconstruction (ART) (see [6]) and maximum entropy (see, for example, [11]) were generally unsuitable for use in this application because of the large noise level. Instead, we have developed a new multivariate reconstruction method. This will be described in Section III.C.2.

Singular Value Decomposition Analysis While impractical for solving (15) due to the large image size and number of measurements, singular value decomposition (SVD) provides insight into the effects of the measurement noise in $V$ on the estimated image (in $S$ ). Consider the use of SVD to compute a least-squares solution to (15). Using SVD to factor $H$,

$$
H=U \Lambda V^{T}
$$

where $U$ is a $N \times N$ orthogonal matrix, $V$ is a $M \times M$ orthogonal matrix, and $\Lambda$ is a $N \times M$ diagonal matrix of singular values.

A least-squares estimate, $\widehat{S}$, of $S$ can be computed as

$$
\widehat{S}=H^{\dagger} Z=H^{\dagger}[H S+\mathcal{V}]
$$

where $H^{\dagger}$ is the Moore-Penrose pseudo-inverse of $H$. Let $\tilde{S}=Z-\widehat{S}$ and $\bar{S}=H^{\dagger} H S$. The least-squares fit error $\tilde{S}$ is

$$
\tilde{S}=\bar{S}-H^{\dagger} \mathcal{V} \text {. }
$$

For $H$ less than full-rank, bias errors in the estimation of $S$ occur; however, if $H$ is full-rank, $H^{\dagger} H=I$ and $S=\bar{S}$ so that

$$
\tilde{S}=H^{\dagger} \mathcal{V}
$$

Hence, the noise $(\tilde{S})$ in the estimates of $\mathcal{A}$ is a linear function of the noise $(\mathcal{V})$ in all the observations in $Z$, even those at some distance from a given resolution element. The inclusion of this additional noise is the price paid for obtaining enhanced resolution.

The effects of the noise can be mitigated to some degree by SVD filtering in which singular values less than a threshold are set to zero. This eliminates the noise effects in the eigenspace spanned by the eigenvectors corresponding to the zeroed singular value but it also adds distortion (see [15]).

Assuming independence of the noise terms in the measurements, the noise covariance matrix $P=E\left[\mathcal{V} \mathcal{V}^{T}\right]$ will be diagonal. In an area of nearly uniform $A, P=\sigma_{v}^{2} I$. From (18) the noise component $\tilde{s}_{i}$ added to the $i^{t h}$ element of the estimate of $S$ will be

$$
\tilde{s}_{i}=\left(H^{\dagger} \mathcal{V}\right)_{i}=\sum_{j} H_{i, j}^{\dagger} \mathcal{V}_{j}
$$

Thus, it follows from the independence of the noise terms that the variance of $\tilde{s}_{i}$ will be

$$
\operatorname{Var}\left[\tilde{s}_{i}\right]=\sigma_{v}^{2} \sum_{j}\left(H_{i, j}^{\dagger}\right)^{2}=\left\|H_{i}^{\dagger}\right\|_{2}^{2} \sigma_{v}^{2}
$$


where $\left\|_{i} H^{\dagger}\right\|_{2}$ is the $L_{2}$ norm of the $i$ th row of $H^{\dagger}$. The normalized standard deviation, $K_{p}$, for the $i^{\text {th }}$ resolution estimate is then

$$
K_{p}(\text { resolution element })=\left\|{ }_{i} H^{\dagger}\right\|_{2} \sigma_{v} / s_{i} .
$$

Since $K_{p}$ for a conventional resolution scatterometer measurement cell over the same area would be (essentially) $K_{p}($ cell $)=$ $\sigma_{v} / s_{i}$, it follows that

$$
K_{p}(\text { resolution element }) \approx\left\|_{i} H^{\dagger}\right\|_{2} K_{p}(\text { cell }) .
$$

The value of $\left\|{ }_{i} H^{\dagger}\right\|_{2}$ depends on a large number of factors including the number and distribution of the measurement cells and the resolution element size; however, based on our empirical observations, $\left\|{ }_{i} H^{\dagger}\right\|_{2}$ is proportional to the ratio of the square of the average number of resolution elements in each low resolution cell and the average number of low resolution cells which cover a given resolution element. Hence, in order to minimize the estimate noise (that is, minimize $K_{p}$ (resolution element)) for a given resolution element size, the number of measurement cells should be maximized. This requires multiple orbit passes. Alternately, the limit in the ultimate resolution is dictated by a combination of the noise level in the measurements and in the measurement overlap: as the resolution element size is reduced, $\left\|{ }_{i} H^{\dagger}\right\|_{2}$ increases, resulting in additional image noise. Thus, the resolution element size must be a tradeoff between the imaging time interval and the image estimate noise level. (We define the "imaging time interval" to be the length of time required to accumulate all the measurements used to generate an enhanced resolution image.)

We also note that if the resolution element size is very small or the overlap in the measurements is insufficient, $H$ may be underdetermined. It follows that there will be multiple $S$ which satisfy (15). To insure a unique estimate of $S$, a maximum entropy-based approach will be used. While this yields a unique solution, the estimate of $S$ may be smoothed, i.e., the effective resolution (defined as the minimum resolvable feature size) may be degraded. Thus, the ultimate resolution is limited by the rank of $H$ and therefore by the measurement cell overlap and the number of measurements. For a fixed number of measurements, reducing the size of the resolution elements arbitrarily will not increase the effective resolution of the resulting estimate but it will increase the estimate noise.

For SASS data, lengthening the imaging time interval increases the number of measurements which also increases the measurement overlap, hence the desire to maximize the imaging time interval to achieve optimum resolution. However, the target characteristics and the instrument calibration must remain constant for a longer period. In this paper we have used the entire 3-month SASS data set to generate images of $\mathcal{A}$ and $\mathcal{B}$ over a test region in the Amazon basin at a resolution of $\sim 3.7 \mathrm{~km}$. For many areas of the extended Amazon basin, satisfactory images can be produced with better resolution and/or shorter time scales.

Reconstruction Approach In order to solve (15) for a general $H$ we have developed a new iterative method based on multiplicative algebraic reconstruction (MART). Conventional single-variable MART can be shown to be a special case of maximum entropy [6]. In MART, each measurement is compared to a predicted (forward projection) value computed from the current image estimate. A multiplicative correction factor is then applied each pixel covered by the measurement causing the forward projection to equal the measurement. Subsequent measurements further alter the pixel values. Over multiple iterations, the correction factors ideally converge to a value of unity and all the forward projections match the measurements. Unfortunately, MART has limited noise tolerance. We have developed a modified form of MART which has been optimized for noisy scatterometer measurements. To estimate both $\mathcal{A}$ and $\mathcal{B}$ the algorithm was further extended to perform multivariate image reconstruction. To simplify presentation of our scatterometer image reconstruction (SIR) algorithm we first provide a brief description of a single variable ( $\mathcal{A}$-only) version of the algorithm.

Estimation of $\mathcal{A}$ Given $\mathcal{B}$. Conventional reconstruction methods provide an iterative solution to (15) in which $\mathcal{V}$ is assumed to be zero (or, equivalently, in which $\mathcal{V}$ is ignored); however, noise tolerance can be increased by using a simultaneous (or, block) update algorithm [6]. In block MART, an initial value image $S^{0}$ (typically a uniform constant) is chosen. In the $k^{t h}$ iteration, each image pixel $s_{i}^{k}$ of the image $S^{k}$ is updated according to

$$
s_{i}^{k+1}=s_{i}^{k} \frac{1}{p_{i}} \sum_{j=1}^{N} h_{j i}\left(\frac{z_{j}}{f_{j}^{k}}\right)^{w}
$$

where $f_{j}^{k}$ is the forward measurement projection, i.e.,

$$
f_{j}^{k}=\frac{1}{q_{j}} \sum_{l=1}^{M} h_{j l} s_{l}^{k},
$$

$p_{i}$ is the number of measurements covering the pixel, and $q_{j}$ is the number of pixels in the measurement, i.e.,

$$
\begin{aligned}
p_{i} & =\sum_{l=1}^{N} h_{l i} \\
q_{j} & =\sum_{l=1}^{M} h_{j l} .
\end{aligned}
$$

The adjustable parameter $w$ is subjectively selected. The case with $w=1$ will be termed unweighted MART while $w=1 / 2$ will be referred to as weighted MART. When applied to the noisy scatterometer data we found that $w=1 / 2$ provided subjectively better images. For convenience, (24) can be written as

$$
s_{i}^{k+1}=\frac{1}{p_{i}} \sum_{j=1}^{N} h_{j i} u_{i j}^{k}
$$

where the update terms $u_{i j}^{k}$ are defined as

$$
u_{i j}^{k}=s_{i}^{k}\left(\frac{z_{j}}{f_{j}^{k}}\right)^{w}=s_{i}^{k} d_{j}^{k}
$$

where

$$
d_{j}^{k}=\left(\frac{z_{j}}{f_{j}^{k}}\right)^{w}
$$


The algorithm is iterated (on $k$ ) until convergence is achieved. Convergence is guaranteed for a consistent system of equations. However, because of the high noise level in the measurements the equations are not consistent and the MART algorithm may not converge or may result in an unsatisfactory solution. To improve the noise tolerance and convergence characteristics, an update scheme which non-linearly weights the measurements and the previous estimates was developed. Equation (28) is used with $u_{i j}^{k}$ redefined as

$$
u_{i j}^{k}= \begin{cases}{\left[\frac{1}{2} \frac{1}{f_{j}^{k}}\left(1-\frac{1}{d_{j}^{k}}\right)+\frac{1}{s_{i}^{k} d_{j}^{k}}\right]^{-1}} & d_{j}^{k} \geq 1 \\ {\left[\frac{1}{2} f_{j}^{k}\left(1-d_{j}^{k}\right)+s_{i}^{k} d_{j}^{k}\right]} & d_{j}^{k}<1 .\end{cases}
$$

This nonlinear update computation limits the size of the update step for large deviations between the forward projection and the measurement. This tends to reduce the effects of noise and aids in convergence.

Estimation of Both $\mathcal{A}$ and $\mathcal{B}$. While the modified block ART algorithm provides satisfactory results for known $\mathcal{B}(c, a)$, in general $\mathcal{B}(c, a)$ is not known and both $\mathcal{A}$ and $\mathcal{B}$ must be estimated. This can be accomplished by modifying the algorithm previously described. We have observed that satisfactory $\mathcal{A}$ images can be generated even when there are small errors in the $\mathcal{B}$ values. This is due to both the small value and the small variation in $\mathcal{B}$. Since the mean value of $\mathcal{B}$ over the Amazon region is approximately 0.13 and $0.05 \leq \mathcal{B} \leq 0.2$ (globally), assuming that $\mathcal{B}=0.13$ yields a worst-case $\mathcal{A}$ estimate error of approximately $1 \mathrm{~dB}$.

A serious difficulty can arise in estimating both $\mathcal{A}$ and $\mathcal{B}$ when the range of incidence angles spanned by the $\sigma^{\circ}$ measurements of a given area is insufficient: we cannot expect to estimate $\mathcal{B}$ with any degree of certainty. Over the study region, generally each resolution element is observed with a wide diversity of incidence angles. For the exceptions, a $\mathcal{B}$ must be determined from other sources in order to correctly estimate $\mathcal{A}$. One logical choice is to use the average of nearby $\mathcal{B}$ estimates. While this may introduce some error in the $\mathcal{A}$ estimate, the error will generally be small. Filling in the missing $\mathcal{B}$ estimate in this way, however, reduces the intrinsic resolution of the $\mathcal{B}$ estimates.

A very simple approach to simultaneous estimation of $\mathcal{A}$ and $\mathcal{B}$ may be derived from the unweighted MART algorithm. For $S_{i}^{0}=$ constant, the first iteration of unweighted MART, $S^{1}$ is

$$
s_{i}^{1}=\frac{1}{p_{i}} \sum_{j=1}^{N} h_{j i} z_{j} .
$$

Remembering that $h_{i j}$ is either one or zero, we see that $s_{i}^{1}$ is the average of the measurements covering the $i^{\text {th }}$ pixel. This averaging approach (which will be referred to as AVE) provides a smoothed initial image estimate which is improved by later iterations. We note that the AVE resolution is better than the measurement cell resolution and is significantly better than the conventional "binning" approach used by Kennett and $\mathrm{Li}[14]$ where the minimum resolution element size is effectively limited to the maximum size of the measurement cells though it may under- or overestimate the image value.
AVE can easily be extended to the multivariate case using our linear model (1): the $\mathcal{A}$ and $\mathcal{B}$ estimates for a given pixel are computed by linear regression of the $\sigma^{\circ}$ measurements (in $\mathrm{dB}$ ) which cover the pixel. This idea forms the basis of the multivariate SIR algorithm.

In the multivariate SIR algorithm, an initial $\mathcal{B}$ estimate image, $B^{0}$, is made by setting $b_{i}^{0}=0.13$ (the global average of $\mathcal{B}$ ). In the $k^{t h}$ iteration of the SIR algorithm, the previous $\mathcal{B}$ estimate image is used to normalize the $\sigma^{\circ}$ measurements from which an estimate of $\mathcal{A}$ is generated using (28) and (31). Linear regression of the update terms (expressed at the measurement incidence angle) is then used to update the previous estimate of $\mathcal{B}$. The new $\mathcal{B}$ estimate is determined as a weighted average of the previous $\mathcal{B}$ estimate and the $\mathcal{B}$ estimate update. The multivariate SIR algorithm is given below.

For processing the SASS data set, we express the measurements $z_{j}$ in $\mathrm{dB}$ so the forward projection $f_{j}^{k}$ is computed in normal space while the $\mathcal{A}$ and $\mathcal{B}$ estimates are in log space, i.e.,

$$
f_{j}^{k}=10 \log _{10}\left[\frac{1}{q_{j}} \sum_{n=1}^{N} h_{j n} 10^{s_{n}^{k} / 10}\right]
$$

where $q_{j i}$ is defined in (27). Define $d_{i j}^{k}$ as (compare (30))

$$
d_{i j}^{k}=\left(\frac{z_{j}-b_{i}^{k}\left(\theta_{j}-40^{\circ}\right)}{f_{j}^{k}}\right)^{w} .
$$

The $\mathcal{A}$ estimate update term, $u_{i j}^{k}$, is computed as in (31),

$$
u_{i j}^{k}= \begin{cases}{\left[\frac{1}{2} \frac{1}{f_{j}^{k}}\left(1-\frac{1}{d_{i j}^{k}}\right)+\frac{1}{s_{i}^{k} d_{i j}^{k}}\right]^{-1}} & d_{i j}^{k} \geq 1 \\ {\left[\frac{1}{2} f_{j}^{k}\left(1-d_{i j}^{k}\right)+s_{i}^{k} d_{i j}^{k}\right]} & d_{i j}^{k}<1\end{cases}
$$

with the $\mathcal{A}$ estimate, $s_{i}^{k}$, updated according to (28) (repeated here for completeness)

$$
s_{i}^{k+1}=\frac{1}{p_{i}} \sum_{j=1}^{N} h_{j i} u_{i j}^{k} .
$$

To compute the $\mathcal{B}$ estimate, let

$$
\begin{aligned}
r_{i} & =\sum_{j=1}^{N} h_{j i} \theta_{j}^{2} \\
t_{i} & =\sum_{j=1}^{N} h_{j i} \theta_{j}
\end{aligned}
$$

and

$$
\zeta_{i j}^{k}=u_{i j}^{k}+b_{i}^{k}\left(\theta_{j}-40^{\circ}\right) .
$$

Then, the linear regression of the $\mathcal{A}$ updates, $u_{i j}^{k}$, provides an update for $\mathcal{B}$,

$$
c_{i}^{k}=\frac{1}{p_{i} r_{i}-t_{i}^{2}}\left(p_{i} \sum_{j=1}^{N} h_{j i} \theta_{j} \zeta_{i j}^{k}-t_{i} \sum_{j=1}^{N} h_{j i} \zeta_{i j}^{k}\right) .
$$

This update is only usable if the range of incidence angles $\left(\theta_{j}\right)$ used in (40) is sufficiently wide. Since a wider incidence angle 
range implies greater confidence in the $\mathcal{B}$ estimate update, the $\mathcal{B}$ estimate is updated using a weighted average of $c_{i}^{k}$ and the previous $\mathcal{B}$ estimate. The weighting factor is a simple function of the variance of $\theta$, i.e., let

$$
x_{i}=\frac{p_{i}}{t_{i}^{2}} \sum_{j=1}^{N} h_{j i} \theta_{j}^{2}-1
$$

then the $\mathcal{B}$ estimate image is updated according to

$$
b_{i}^{k+1}=\frac{1}{x_{i}+1}\left(x_{i} c_{i}^{k}+b_{i}^{k}\right) .
$$

This system of equations is iterated over $k$ until convergence. Unlike conventional MART which diverges if the iteration is continued too long, the SIR algorithm does not diverge after continued iteration. Instead, as the algorithm converges the update terms continue to grow smaller until there is no change between iterations. The algorithm has been tested extensively using simulated and actual SASS measurements and typically converges within 30-50 iterations.

We observed that the subjective quality of the final images may be improved by applying an edge-preserving $3 \times 3$ median filter to the $\mathcal{A}$ and $\mathcal{B}$ images. It was further observed that the image noise can be significantly reduced, with only a small reduction in the image resolution, by application of a hybrid median-linear filter to the image estimates at each stage of the iteration. The SIR algorithm with the added filtering will be termed SIRF.

In the hybrid filter, the image values within a moving $3 \times 3$ window are ordered and the median determined. If the difference between the second highest and second lowest values within the window is less than 0.25 , the center pixel is replaced with the average of the middle seven values of the ordered window pixels. Otherwise, the center value is replaced with the median value. Effectively, this hybrid filter acts like a linear filter when the algorithm is near convergence and the region is smooth but operates as a median filter otherwise. The edge-preserving properties of the median filter maintain resolution while providing noise suppression.

\section{Simulation Results}

To initially evaluate our technique we used simulated SASS $\sigma^{\circ}$ measurements of a synthetic scene. The synthetic scenes $(\mathcal{A}$ and $\mathcal{B})$, shown in Fig. 7 , were designed to evaluate the accuracy and the effective resolution of the estimated images. They include a number of features broadly similar to those expected in the actual Amazon basin, including 1) large expanses of nearly constant $\mathcal{A}$ and $\mathcal{B}, 2$ ) slow gradients in $\mathcal{A}$ and $\mathcal{B}, 3$ ) sharp boundaries in $\mathcal{A}$ alone, $\mathcal{B}$ alone, or in $\mathcal{A}$ and $\mathcal{B}$ simultaneously, and 4 ) sinuous, small-scale "rivers" which have smaller $\mathcal{A}$ than the surrounding areas. While actual rivers and bodies of open water will have somewhat variable $\sigma^{o}$ due to the effect of wind roughening the surface, they will appear significantly darker than the surrounding vegetation. In our synthetic image, the "rivers" have a constant $\sigma^{\circ} . \sigma^{o}$ measurements over large bodies of water and the ocean are not used.

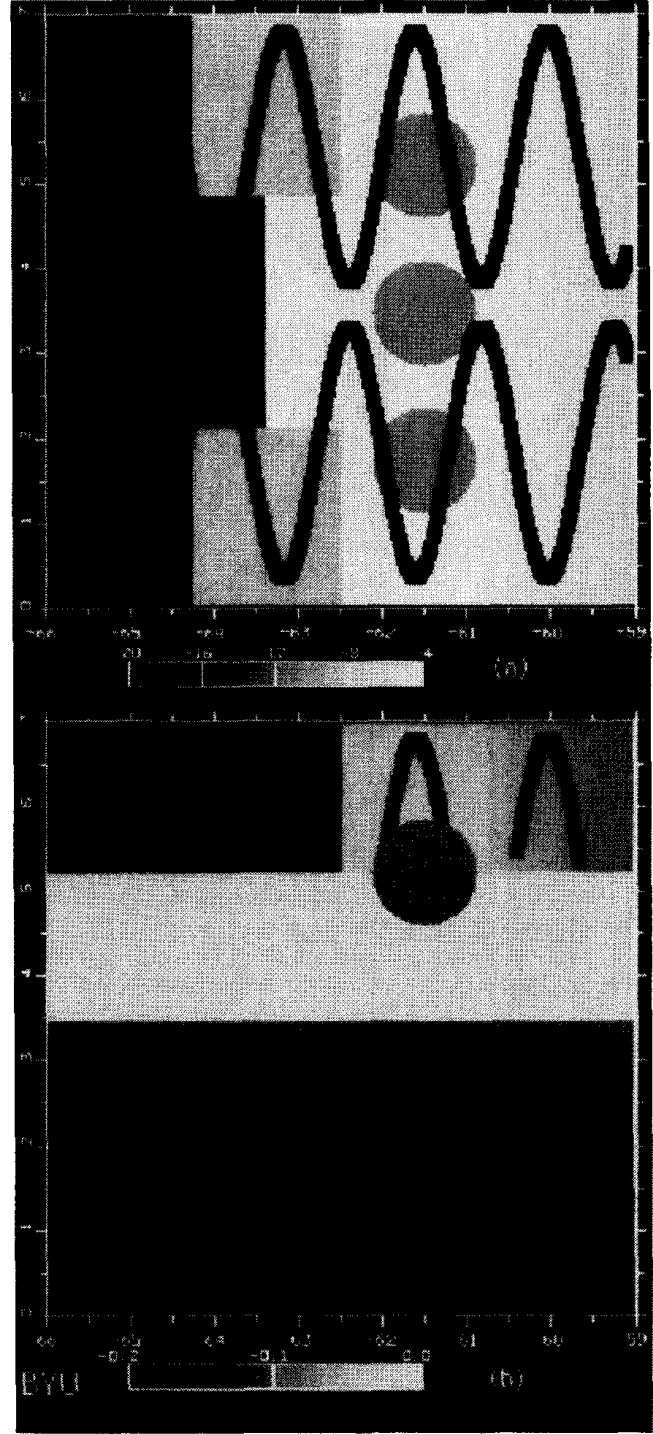

Fig. 7. (a) Synthetic $\mathcal{A}$ image with various test targets. (b) Corresponding synthetic $\mathcal{B}$ image.

The synthetic scene covers a $7^{\circ}$ latitude by $7^{\circ}$ longitude corresponding to a portion of the Amazon basin (refer to Fig. 8). To generate the simulated $\sigma^{\circ}$ measurements, actual SASS measurements covering this region were used. The SASS measurement cell geometry and the $K_{p}$ were extracted from the SASS SDR files (only vertically polarized measurements were used). The $\sigma^{o}$ measurement value was then replaced with the value computed from the synthetic scene using the cell geometry. To this was added Monte Carlo Gaussian noise with variance computed from the synthetic $\sigma^{\circ}$ value and the $\alpha, \beta$, and $\gamma$ coefficients derived from the actual $K_{p}$ (see (4)) in the SASS SDR. Fig. 9 illustrates typical cell measurement areas for each beam at the near swath (near the nadir track) and far swath for ascending (north-bound) and descending (south-bound) spacecraft passes. The relative sizes and the 


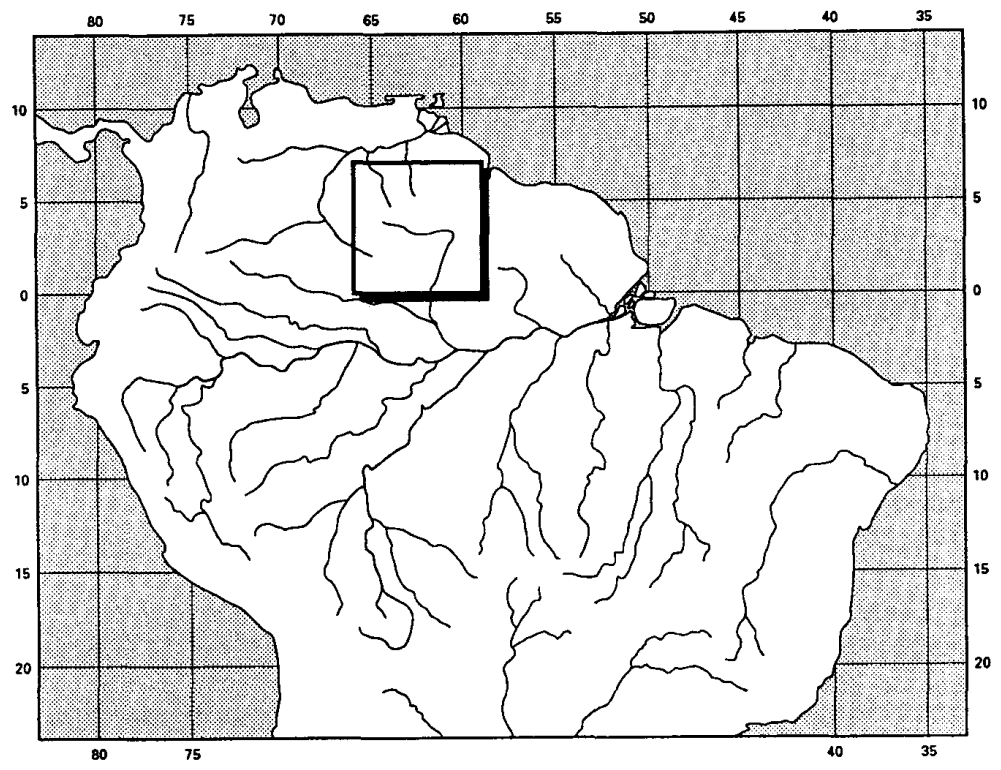

Fig. 8. Location map of the $7^{\circ}$ by $7^{\circ}$ test region in the Guiana Highlands of South America.

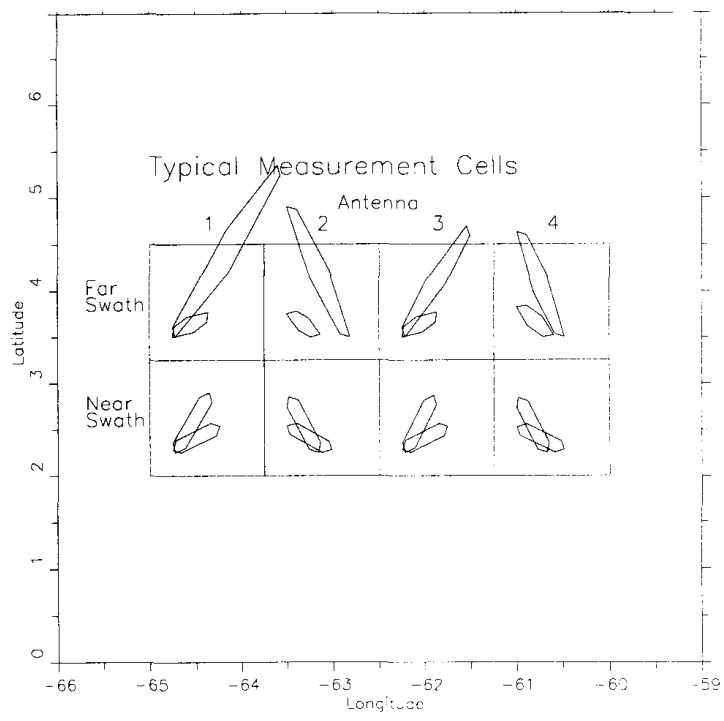

Fig. 9. Typical cell geometries for measurement cells in the near and fa swath for each antenna beam. Both ascending (north-bound) and descending (south-bound) spacecraft passes over the test region are illustrated. The cells are shown at the same scale as the images to follow.

compression and elongation of the cells for different beams and orbit passes is apparent.

Using the SIR and SIRF algorithms described above, $\mathcal{A}$ and $\mathcal{B}$ images were estimated from the simulated $\sigma^{o}$ measurements. The estimated images are shown in Fig. 10. The resolution elements in these images are $1 / 30^{\circ} \times 1 / 30^{\circ}$ (roughly $3.7 \times 3.7$ $\mathrm{km})$. For comparison, images generated using AVE and the "binning" approach $\left(0.5^{\circ} \times 0.5^{\circ}\right.$ resolution $)$ are included in Fig. 10. The resolution enhancement of the SIRF, SIR, and AVE algorithms relative to the "binning" approach is readily appar- ent. The SIRF and SIR provide further resolution enhancement over the AVE images even though some smoothing and noise texturing is evident. In particular, although abrupt transitions in the $\mathcal{A}$ images have been smoothed, fine detail such as the "rivers" remains evident, albeit "fuzzy." Smooth regions and gentle transitions are accurately estimated with minimal error even in regions in which the $\mathcal{B}$ estimate quality is poor. The SIR and SIRF $\mathcal{B}$ images have much less small-scale detail with more smoothing of sharp transitions. However, as expected, these errors appear to have minimal effect on the $\mathcal{A}$ image. The SIRF and SIR images are similar though the SIRF image is somewhat smoother due to the filtering. The difference between these algorithms is more apparent with the actual data (below). Note that in this artificial simulation, both the range and the transition sharpness in $\mathcal{A}$ and $\mathcal{B}$ are more severe than that expected in the actual data. However, the measurement noise in the actual measurements appears to be larger than predicted by the $K_{p}$ value contained in the SDR file.

\section{ACTUAL SASS Data RESUlTS}

To test our technique using real data, we selected a test area over Guiana Highlands which includes portions of Northern Brazil and Southern Venezuela (refer to Fig. 8). A wider view of the Amazon basin is given in the companion paper [18].

The SIR and SIRF algorithms were used with actual SASS vertically polarized $\sigma^{o}$ measurements to generate enhanced resolution maps of $\mathcal{A}$ and $\mathcal{B}$ over the test region. The resulting image estimates are shown in Fig. 11. As in the simulated case, the resolution elements are $1 / 30^{\circ} \times 1 / 30^{\circ}$. Images generated using AVE and the "binning" approach are given for comparison. The imaging time interval was the entire 3-month SASS mission. Orbits (revs) for which the measurements exhibited excessive error were not included (see [4] for a detailed description of the SASS data anomalies and the 


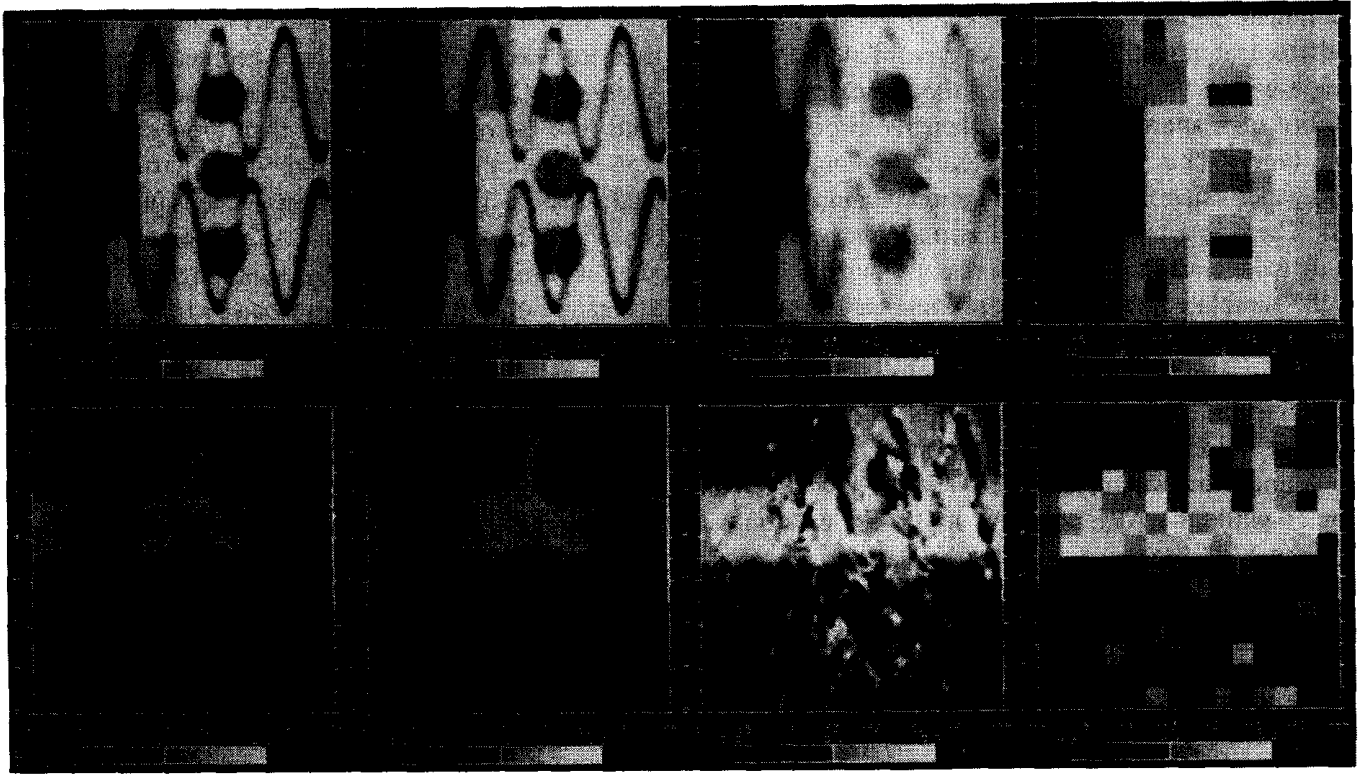

Fig. 10. (a) High resolution $\mathcal{A}$ estimate image generated from simulated SASS using the SIRF algorithm. The resolution elemen size is $1 / 30^{\circ} \times 1 / 30^{\circ}$ (approximately $3.7 \times 3.7 \mathrm{~km}$ ). (b) $\mathcal{B}$ estimate image corresponding to a. (c) $\mathcal{A}$ estimate image generated using the SIR algorithm. (d) $\mathcal{B}$ estimate image corresponding to $\mathrm{c}$. (e) $\mathcal{A}$ estimate image generated using the AVE algorithm. (f) $\mathcal{B}$ estimate image corresponding to e. $(\mathrm{g}) 0.5^{\circ}$ (approximately $55 \mathrm{~km}$ ) resolution $\mathcal{A}$ image generated from the same simulated SASS measurements using the "binning" approach. (h) $\mathcal{B}$ image corresponding to $\mathrm{g}$
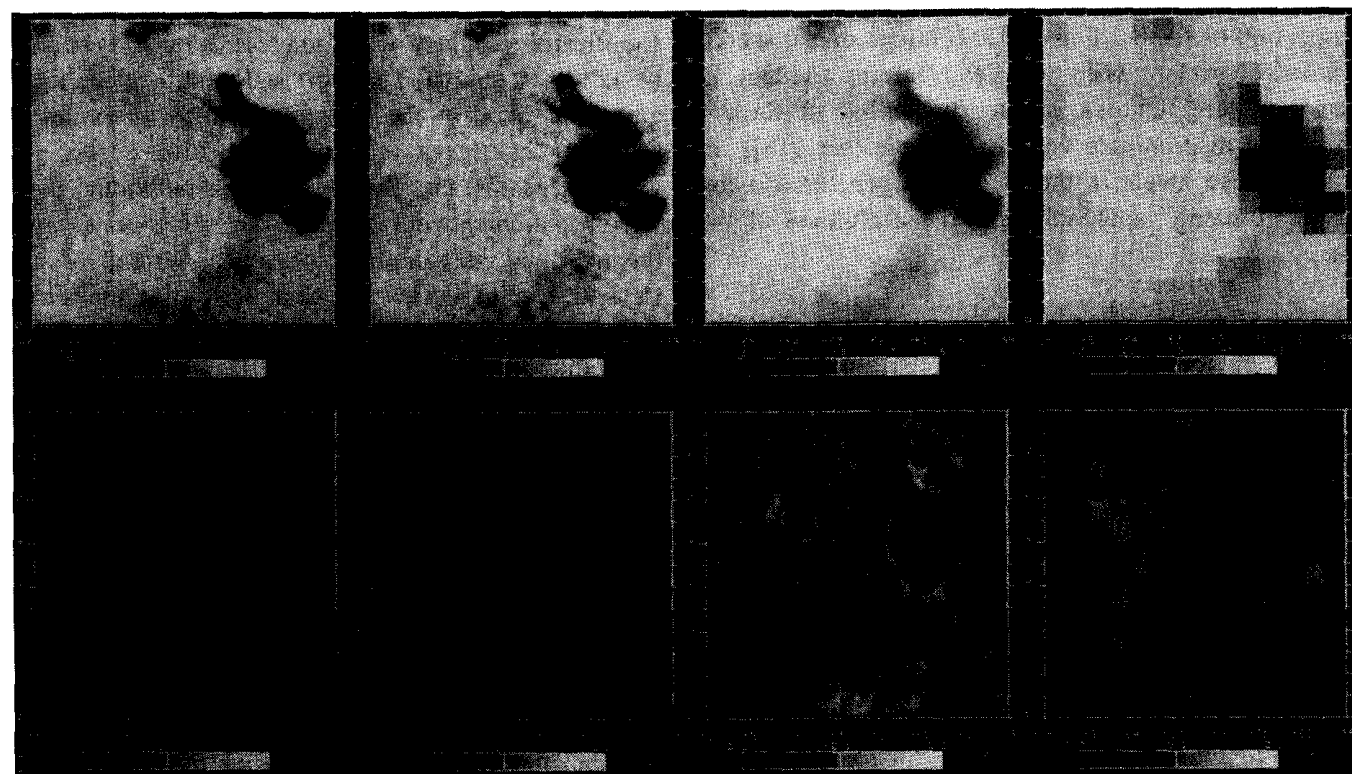

Fig 11. (a) High resolution $\mathcal{A}$ image of the Guiana Highlands reconstructed from actual SASS data (vertical polarization) using the SIRF algorithm. The resolution element size is $1 / 30^{\circ} \times 1 / 30^{\circ}$ (approximately $3.7 \times 3.7 \mathrm{~km}$ ). (b) $\mathcal{B}$ estimate image corresponding to a. (c) $\mathcal{A}$ estimate image generated using the SIR algorithm. (d) $\mathcal{B}$ estimate image corresponding to c. (e) $\mathcal{A}$ estimate image generated using the AVE algorithm. (f) $\mathcal{B}$ estimate image corresponding to e. (g) $0.5^{\circ}$ (approximately $55 \mathrm{~km}$ ) resolution $\mathcal{A}$ image generated from the same actual SASS measurements using the "binning" approach. (h) $\mathcal{B}$ image corresponding to $\mathrm{g}$.

rationale for selecting and excluding orbits with excessive error).

The low $\mathcal{A}$ (black and dark gray) area in Fig. 11 corresponds to tall grassland (black) with broadleafed evergreen trees and shrubs (dark gray). An area of tropical forest immediately north of the dark region appears as shades of light gray on the image. Smaller areas of lowland forest also appear as dark areas along the north-central portion of the image. 
The predominate light area corresponds to very moist multicanopied tropical forest. The high degree of correlation of the $\mathcal{A}$ image with a vegetation map of the same area hints at the capability of scatterometer measurements to discriminate vegetation types. This topic is explored at length in the companion paper using images of the entire extended Amazon basin [18]. There is a high degree of correlation between the observed $\mathcal{A}$ value and the vegetation canopy density.

As in the simulated case, the resolution enhancement in the SIRF, SIR, and AVE algorithms relative to the "binning" approach are clearly evident in the $\mathcal{A}$ images in Fig. 11. Using the actual measurements, however, the tradeoff between resolution and noise is more readily apparent. In particular, the SIRF $\mathcal{A}$ image has significantly less "noise" than does the SIR $\mathcal{A}$ image while the AVE $\mathcal{A}$ image looks like a smoothed version of the SIRF image.

In an effort to explain the difference in performance between the SIRF and SIR algorithms for simulated and actual data, we increased the noise level (i.e., increased the $K_{p}$ ) of the simulated measurements. When the noise level of the simulated measurements is increased by $10 \%$ or more, the relative performance of the SIR and SIRF algorithms resembles the performance of the algorithms when applied to the actual SASS measurements. Apparently, the actual $\sigma^{\circ}$ measurements contain higher noise levels than indicated by the SDR $K_{p}$ value, possibly due to unmodeled noise sources.

The $\mathcal{B}$ images show only limited spatial detail; however, careful inspection of the SIRF and SIR $\mathcal{B}$ image reveal spatial features which correlate with the key features evident in the $\mathcal{A}$ image. These features add to the ability of the $\sigma^{\circ}$ measurements to discriminate vegetation types (see [18]). In contrast, high noise levels in the $\mathcal{B}$ images generated using AVE and the "binning" approach limit the usefulness of the $\mathcal{B}$ images.

\section{APPLICATION TO FUTURE SCATTEROMETERS}

As presented here, the scatterometer resolution enhancement is based on measurement cell overlap. For SASS, overlap was obtained by combining multiple orbits over an extended period of time. Increasing the number of orbits included in the image reconstruction results in improved resolution and reduced estimate noise. However, the target's radar characteristics must remain constant over the imaging time interval. This represents a serious limitation for rapidly changing regions such as polar ice.

For future Doppler scatterometers such as NSCAT and the planned NSCAT-class EoS-era scatterometer, significant resolution enhancement resulting from measurement overlap can be obtained by modifying the measurement sequence. This permits enhanced resolution imaging with only a single spacecraft pass, relaxing the target constancy requirement. The required instrument modifications, described below, are relatively minor.

The $25 \mathrm{~km}$ resolution NSCAT instrument system is described in detail in [19]. Like SASS, an NSCAT-class scatterometer obtains resolution by the combination of a narrow antenna pattern and the timing of transmit pulses. Unlike
SASS, which used fixed-frequency bandpass filters for Doppler filtering, the NSCAT-class scatterometer uses a digital FFT processor [16]. In this processor the radar return echo is split into four channels which are then processed by an FFT into 256 frequency "bins" (effectively very narrow bandpass filters) and squared (power detection). The squared FFT bins are known as periodogram bins. A $25 \mathrm{~km}$ resolution cell power measurement is obtained by summing the bin powers over the range of bins which correspond to the desired bandwidth of the $\sigma^{\circ}$ cell. This process is known as "cell binning." Only the accumulated powers for each of $25 \sigma^{\circ}$ cells/beam are downlinked to the ground. While the periodogram bin frequency resolution (i.e., Doppler bandwidth) is different for each channel, each periodogram bin bandwidth corresponds to approximately $2.5-3.5 \mathrm{~km}$ along-beam distance on the ground.

The narrow antenna illumination pattern defines the crossbeam resolution which varies from approximately $7 \mathrm{~km}$ at the near swath to $15 \mathrm{~km}$ at the far swath. Instrument timing provides the along-track resolution. As currently envisioned for NSCAT, $\sigma^{\circ}$ measurements are made from each of the eight antenna beams in sequence during the time required for the spacecraft to travel $25 \mathrm{~km}$ (approximately $3.68 \mathrm{~s}$ ). For each beam, 25 transmit pulse/receive cycles constitute the signal+noise measurement. Four additional "pulses" in which a transmit pulse is not issued are used to obtain the noise-only measurement.

By making simple modifications to the NSCAT design, significantly higher resolution measurements can be made using our technique. The proposed modifications to the design are to 1) modify the beam-sequential operation described above to interleave the transmit/receive cycles among the different beams, and 2) downlink the individual periodogram bin measurements for further processing on the ground. In the modified measurement sequence, instead of making 25 transmit/receive cycles and four noise-only measurements on a single beam before switching to the next beam, the cycle is modified to transmit a single transmit pulse (or make a single noise-only measurement) on a given beam and then switch to the next beam, cycling through all eight beams in sequence before returning to the first beam for its next transmit pulse. Between the individual transmit pulses on a given beam, the spacecraft will have moved approximately $860 \mathrm{~m}$ (refer to Fig. 12). Hence, the periodogram bin measurements will be on $860 \mathrm{~m}$ centers. They will be $2.5-3.5 \mathrm{~km}$ wide in the along-track direction and so have significant overlap in the along-track dimension. The periodogram bins, though wider in the cross-track dimension $(7-15 \mathrm{~km})$, will have a similar amount of overlap in the cross-track dimension.

To further improve the resolution for imaging over land/ice, the antenna beam sequence can be changed to operate on only a single antenna beam. In this case, the along-track spacing between periodogram bins is reduced to approximately $110 \mathrm{~m}$, resulting in more measurements with more overlap between the measurements and, hence, improved ultimate resolution. This cannot be done for wind measurement over the ocean, since multiple azimuthal measurements of $\sigma^{o}$ are required to retrieve the wind. With the modifications described above the ultimate resolution of the scatterometer with the 


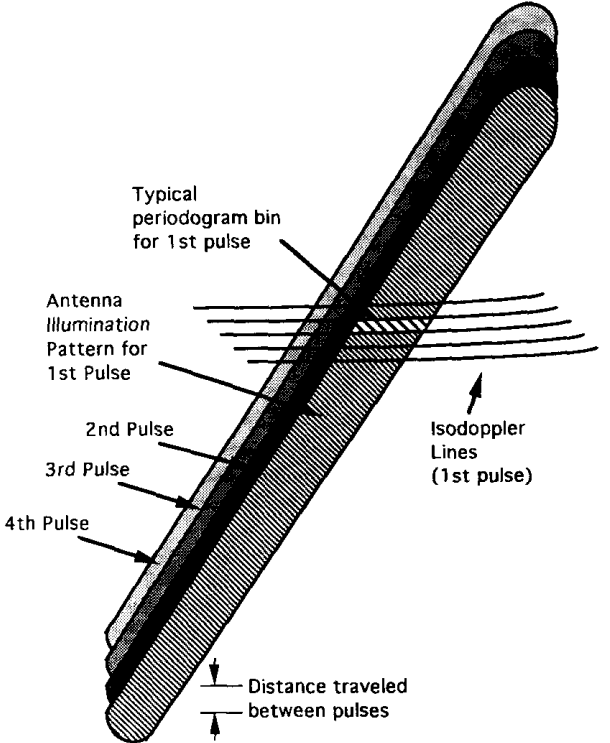

Fig. 12. Diagram illustrating the $\sigma^{o}$ measurement scheme of the proposed high resolution scatterometer technique for an NSCAT-class scatterometer (see text).

ground processing is estimated to be approximately $1-2 \mathrm{~km}$ over land/ice (single beam operation) and $5-10 \mathrm{~km}$ over the ocean (multiple beam operation). The high noise level of the periodogram bin measurements is the primary limitation in ultimate resolution.

The changes discussed above affect only the NSCAT digital subsystem. Even after modification, $25 \mathrm{~km}$ resolutioncompatible measurements can still be made; however, we note that, if the periodogram data is collected continuously, the onboard cell "binning" can be eliminated and can be performed more accurately on the ground. A possible implementation of the proposed changes is to maintain the existing $3.2 \mathrm{kbps}$ $25 \mathrm{~km}$ resolution interface and add a higher rate $(\sim 700$ $\mathrm{kbps}$ ) interface for the periodogram bin data. A scheduled "targets of opportunity" approach can be used for collecting the periodogram bin data while minimizing the downlink data volume.

\section{APPLICATIONS FOR ENHANCED RESOLUTION KU-BAND SCATTEROMETRY}

Although the best obtainable resolution with this method is significantly better than the intrinsic resolution of the scatterometer it remains only medium-scale; however, we believe, based on our preliminary studies, that the resulting mediumscale resolution radar images have significant potential in many areas. In the following subsection we consider some potential applications in land, ice, and wind measurement.

\section{A. Land Imaging}

While $K u$-band $(14 \mathrm{GHz})$ radar signals have very little surface penetration, $K u$-band $\sigma^{\circ}$ is very sensitive to vegetation, surface water, and land surface type. In a separate paper [18] we demonstrate the utility of the scatterometer data for discriminating between broad vegetation groups. Given the very high accuracy of this classification, it may be possible to make precise areal measurements of the global tropical rainforest extent from SASS data taken in 1978. Such measurements can then be compared with measurements from NSCAT in 1995 to evaluate tropical deforestation. Similar studies may also be possible for land use and the study of desertification (see, for example, the discussion of the Sahara desert in [13]). The greater resolution possible with suitably modified future scatterometers will permit more detailed studies. The frequent, broad-area coverage afforded by the scatterometer may also allow for daily vegetation and surface water monitoring as well as studies of diurnal variation of vegetation over large regions.

\section{B. Ice Imaging}

Carsey [5] and Drinkwater [7], [8] have extensively studied the radar response of ice and snow. Their results indicate that $\sigma^{\circ}$ can be used to map new ice, snow cover, and the seasonal variation in the ice edge. At $K u$-band, the scatterometer measurements are particularly sensitive to ice snow cover. Measurement of snow cover is considered crucial to understanding the ice/air heat flux of the polar regions. Snow cover data at $1-2 \mathrm{~km}$ resolution from future scatterometers, coupled with radiometer measurements, will permit significant improvements in the understanding of this critical heat flux component of the global energy budget. In addition, 1-2 $\mathrm{km}$ resolution may be sufficient for the study of near-ice edge oceanic eddies and upwelling phenomena (Carsey and Drinkwater, personal communications).

A scatterometer with the proposed modifications would provide a good measurement platform for polar ice data since it provides 1) frequent coverage and 2) multiple incidence angle observations. From the latter it may be possible to infer ice-age as well as snow cover and ice motion. We are currently investigating the use of our technique for ice studies of Greenland. Preliminary results dramatically illustrate the capability of the scatterometer to monitor the extent of the summer melt.

\section{High Resolution Wind Measurement}

While we have concentrated on land/ice imaging in this paper, our technique could also be applied for oceanic wind measurement using future, modified scatterometers. Because of the high variability of the ocean and the orbit sampling of the scatterometer, global measurement of high (better than $25 \mathrm{~km}$ ) resolution winds may not be appropriate; however, "target of opportunity" diagnostic studies of interesting meteorological conditions can be used to improve local forecasts and for mesoscale studies of air/sea interaction with a resolution of $5-10 \mathrm{~km}$. Because $\sigma^{\circ}$ is generally much lower over the ocean, the effective swath width of the high resolution winds would be smaller than the conventional 25 $\mathrm{km}$ resolution measurements. We note that the conventional resolution measurements can be obtained simultaneously with the high resolution measurements. The high resolution wind 
measurements can be useful in understanding mesoscale and submesoscale atmospheric wind motions and fluxes, especially for focused studies such as the Tropical Ocean Global Atmosphere (TOGA) experiment.

\section{CONCLUSION}

Traditionally, spaceborne scatterometers have been low resolution radar instruments designed to measure winds over the ocean. Because of their low resolution, the scatterometer measurements made over land have been used primarily to calibrate the instrument. However, studies of scatterometer measurements over land have hinted at their sensitivity to vegetation and ice coverage. Improved resolution would enhance the scientific utility of the scatterometer data for land and ice studies.

In this paper we have described a method for obtaining enhanced resolution radar images from scatterometer measurements. The technique is based on spatial measurement overlap and a new image reconstruction algorithm which can tolerate high noise levels. To demonstrate the resolution enhancement of our imaging technique, we have used both simulated and actual SASS data. Resolution enhancement of 8-10 times was achieved. The resulting medium-scale resolution is expected to be useful in a variety of other wide area geophysical studies such as the vegetation classification, ice, and wind measurement.

The limitations of our technique when applied to SASS data may be ameliorated for future Doppler scatterometers such as NSCAT and the NSCAT-class EoS-era scatterometer. With relatively inexpensive modifications, future NSCAT-class scatterometers can achieve effective resolutions as low as 1-2 $\mathrm{km}$ with a moderate increase in downlink telemetry bandwidth. Thus, the modified scatterometer can provide significant additional science return with only a limited budgetary impact. This relatively inexpensive enhancement of planned missions may yield significant contributions to future studies of global change.

While our technique was developed expressly for spaceborne scatterometer measurements (SASS in particular), it can be applied to measurements from other sensors such as radiometers.

\section{ACKNOWLEDGMENT}

We wish to acknowledge the support of the NASA Headquarters programs in Physical Oceanography (David Adamec) and Polar Sciences (Robert Thomas). We also would like to thank Gary Skouson for his help in preparing the SASS data. The SASS SDR data was obtained from the NASA Ocean Data System at the Jet Propulsion Laboratory/California Institute of Technology.

\section{REFERENCES}

[1] I. J. Birrer, E. M. Bracalente, G. J. Dome, J. Sweet, and G. Berthold " $\sigma \circ$ Signature of the Amazon Rainforest obtained from the Seasat scatterometer," IEEE Trans. Geosci. Remote Sensing, vol. GE-20, pp. 11-17, 1982.

[2] D. H. Boggs, "Seasat Algorithm Development Facility Scatterometer Sensor Algorithm Specifications," Seasat Doc. 622-231, Jet Propulsion Laboratory, Pasadena, CA, May 1981.
[3] E. Bracalente, D. Boggs, W. Grantham, and J. Sweet, "The SASS Scattering Coefficient $\left(\sigma_{o}\right)$ Algorithm," IEEE J. Oceanic Eng., vol. OE-5, pp. 145-154, April 1980.

[4] E. Bracalente and J. Sweet, "Analysis of normalized radar cross section $\left(\sigma_{\circ}\right)$ signature of Amazon Rain Forest using Seasat scatterometer data," NASA Tech. Memo. 85779 , NASA NTIS, 1984.

[5] F. D. Carsey, "Summer Arctic sea ice character from satellite microwave data," J. Geophys. Res., vol. 90, no. C3, pp. 5015-5034, May 20, 1985.

[6] Y. Censor, "Finite series-expansion reconstruction methods," Proceedings of the IEEE, vol. 71, pp. 409-419, Mar. 1983.

[7] M. R. Drinkwater and G. B. Croker, "Modelling changes in the dielectric and scattering properties of young snow-covered sea ice at GHz frequencies," J. Glaciology, vol. 34, no. 118, pp. 274-282, 1988.

[8] M. R. Drinkwater, " $K_{u}$ band airborne radar altimeter observations of marginal sea ice during the 1984 Marginal Ice Zone Experiment," $J$. Geophys. Res., vol. 96, no. C3, pp. 4555-4572, March 1991.

[9] R. E. Fischer, "Standard deviation of scatterometer measurements from space," IEEE Trans. Geosci. Electronics, vol. GE-10, pp. 106-113, 1972.

[10] R. Gordon, "A tutorial on ART," IEEE Trans. Nuclear Sci., vol. NS-21, pp. 78-93, June 1974.

[11] E. T. Jaynes, "On the rationale of maximum-entropy methods," Proceedings of the IEEE, vol. 70, no. 9, Sept. 1982

[12] J. W. Johnson, L. A. Williams, Jr., E. M. Bracalente, F. B. Beck, and W. L. Grantham, "Seasat-A Satellite Scatterometer Instrument evaluation," IEEE J. Oceanic Eng., vol. OE-5, pp. 138-144, April 1980.

[13] R. G. Kennett and F. K. Li, "Seasat over-land scatterometer data, Part I: Global overview of the $K u$-band backscatter coefficients," IEEE Trans. Geosci. Remote Sensing, vol. 27, pp. 592-605, Sept. 1989.

[14] R. G. Kennett and F. K. Li, "Seasat over-land scatterometer data, part II: Selection of extended area land-target sites for the calibration of spaceborne scatterometers," IEEE Trans. Geosci. Remote Sensing, vol. 27, pp. 779-788, Nov. 1989.

[15] S-S. Kuo and R. J. Mamone, "Image reconstruction by convex projections using adaptive constraints and the $L_{1}$ norm," IEEE Trans. Signal Processing, vol. 40, pp. 159-168, Jan. 1992.

[16] D. G. Long, C-Y Chi, and F. K. Li, "The design of an onboard digital Doppler processor for a spaceborne scatterometer," IEEE Trans. Geosci. Remote Sensing, vol. 26, pp. 869-878, Nov. 1988.

[17] D. G. Long and J. M. Mendel, "Identifiability in wind estimation from wind scatterometer measurements," IEEE Trans. Geosci. Remote Sensing, vol. 29, pp. 268-276, 1991.

[18] D. G. Long and P. J. Hardin, "Vegetation studies of the Amazon Basin using enhanced resolution Seasat scatterometer data," IEEE Trans. Geosci. Remote Sensing, in press, 1993.

[19] F. M. Naderi, M. H. Freilich, and D. G. Long, "Spaceborne radar measurement of wind velocity over the ocean-an overview of the NSCAT Scatterometer System," Proceedings of the IEEE, vol. 79, pp. 850-866, June 1991.

[20] R. H. Stewart, "Seasat Success Statement," Jet Propulsion Laboratory Publication D-2841, Pasadena, CA, April 11, 1985.

[21] A. Sobti, R. K. Moore, and F. T. Ulaby, "Backscatter response at 13.9 $\mathrm{GHz}$ for major terrain types as seen from orbit," Tech. Rep. 243-4, NASA Lyndon B. Johnson Space Center, Houston, TX, Aug. 15, 1975.

[22] F. T. Ulaby, R. K. Moore, and A. K. Fung, Microwave Remote Sensing-Active and Passive, Vols. 1 and 2. Reading, MA: AddisonWesley Publishing Co., 1981.

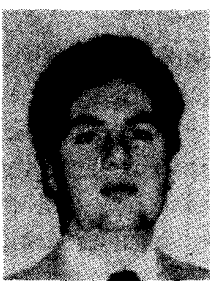

David G. Long (S'80-M'82-S'82-M'83-S'84-M '89) received the B.S. and M.S. degrees in electrical engineering from Brigham Young University, Provo, UT, in 1982 and 1983, respectively, and the Ph.D. degree in electrical engineering from the University of Southern California, Los Angeles, in 1989.

He has been with the Jet Propulsion Laboratory, Pasadena, CA, as a Radar Systems Engineer, since 1983. He has been the Project Engineer for the NASA Scatterometer (NSCAT) project and the Experiment Manager for the Eos SCANSCAT. NSCAT is a spaceborne scatterometer designed to measure oceanic winds from space. NSCAT is planned for flight in 1995 aboard the Japanese Advanced Earth Observing System (ADEos). SCANSCAT is a developmental scatterometer being studied for flight on NASA's Earth Observing System (Eos) in the late 1990's. He was responsible for high-level design, anlaysis, and technical management of these projects to insure that the projects meet all mission requirements. 
His responsibilities included overseeing instrument design and fabrication, algorithm, and coding for the ground processing system, mission operations, and data analysis. His technical responsibilities included system performance analysis, high-level design, development and maintenance of system requirements, and supervision of the Systems Engineering staff. He is an Assistant Professor in the Electrical and Computer Engineering Department at Brigham Young University. His research interests include computer graphics, speech and signal processing, estimation theory, radar, and mesoscale atmosphere dynamics.

Dr. Long has received the NASA Award of Achievement several times.
Perry J. Hardin (M'92) photograph and biography not available at the time of publication.

Peter T. Whiting, photograph and biography not available at the time of publication. 